\section{A Materials Society}

A recent newsletter of the Forum on Physics and Society of the American Physical Society carried an article entitled "Physics and Society: Not Subjects Apart" by Albert A. Bartlett (University of Colorado). ${ }^{\prime}$ It is based on a talk given by Bartlett a year earlier at an APS meeting. His central theme is that where pre-World War II undergraduate physics courses included the relevance of the subject to the real world, modern courses are tuned to the few students who will ultimately earn physics doctorates, focusing on the discipline itself without connecting it to our daily lives. Bartlett ascribes a decline in the number of physics majors and the public's lack of appreciation for physics to this educational trend. Noting that it was World War I that brought chemistry to prominence, he continues, "World War II was the war in which physics exploded into prominence." ${ }^{2}$ Physics education has, however, failed in recent times to capitalize on that visibility. He laments that physics' drive to reproduce itself, mitigates against diversity, and favors, according to an ecological analogy, its eventual demise.

To place all the blame on the educators may be going a bit too far. Beyond the control of the planners of curricula and course content is the fact that some concepts such as chemicals and chemistry relate more directly to tangible and visible aspects of daily life. It is no accident that well-known corporations include "chemical" in their names, but no product oriented "physics" companies come to mind. Since WWII there is also a new, less traditional, player on the field. Materials are here!

We can't say that the relevance of physics to all societal issues (especially the immaterial, that is the more philosophical ones) is now handled by materials science and technology. But we can propose that the abdication of physics from the role of introducer of new high-tech materials into everyday use has left a vacuum filled by the multidisciplinary materials field. We agree, without invoking the ecological analog, that the diverse culture is the one that thrives-and no science is more diverse than materials. Other classical disciplines have followed a path similar to that of physics. As math departments eschewed computer science and polymers were evicted from many chemistry departments, the parent disciplines retreated to the ivory tower and their progeny coalesced to what we can view as the connection of science (including physics) to society.

Bartlett recognizes this trend when he says, "the applied topics that (physics) did not deign to offer were discarded." Indeed they were-by physics-but they were retrieved by what is now called materials science. Physics may return to teach us all how the moon orbits the earth, how fusion brings us the energy of the sun, or why things fall down instead of up. But, the train has left the station with the microcircuit and optical discs, the VCR and time-release capsules, and yes, even the practical superconductor.

Does this mean that the onus is now on materials scientists to explicitly maintain the link between the science and the society? Not really. Let us propose that what has really happened is that the thorough mixing of disciplines in modern technology has rendered the boundaries between fields more diffuse. Physics, for example, may indeed be considered the parent of at least part of what we call materials science. It needed, however, to be mated to pieces of chemistry, metallurgy, engineering, etc., to form an integrated field. Physics remains at the basic end of the spectrum supplying the most fundamental understanding, but science as a whole bears the responsibility to relate itself to societal issues and needs.

Symptomatic of the transition to less distinct inter-field borders are the skirmishes and incursions along these borders which seem to arise from a desire to cling to older, more comfortable definitions and to defend the "home" discipline. On occasion physics may be seen reclaiming previously ceded territory. The National Academy survey, ${ }^{3}$ Physics Through the 1990s, views materials science as a physics subdiscipline and labels notable achievements such as solid state chemistry's discovery of the new high $T_{c}$ superconductors as an advance in physics. Of course it serves no practical purpose to quibble about who has disciplinary ownership. The condensed matter theorists will sooner or later tell us what factors make such materials behave as they do and all the materials R\&D fields will contribute to bringing such advances to society. Jockeying for the most advantageous position can also be seen in the broadened programming and renaming of disciplinary technical societies where, with no significant change in membership or constituency, fields of interest have been redefined to match more multitopical materials pursuits.
In times of limited funds and a perceived "zero-sum game" budget algorithm, it may be natural for each constituency to attempt to maximize its own share by claiming a central role in visible and high leverage activities. (It would not be surprising if the soon-to-be completed Academy study on Materials Science \& Engineering claimed physics as a subdiscipline and assumed ownership of such as high $T_{c}{ }^{\prime} s$.) As reported many places, including recently the MRS BULLETIN, those knowledgeable in the federal funding process remind us that internal squabbles among scientific interest groups damage everyone's prospects.

Rather than bemoan the distancing of physics specialties per se from public recognition and direct societal impact, attention could be paid to public relations and perceptions of science and engineering in general. A healthy technical community is necessary for the economic well being of our technological society. The public needs to view science in that light without distinguishing among fields. Although Bartlett's narrow focus on physics education might need broadening in view of today's fuzzier disciplinary distinctions, the essence of his conclusion is easily extrapolated to the broader context. He states, "It is essential that students in our introductory courses be treated, not as potential physics majors, but as active interdisciplinary participants and leaders in a society of educated people." Hear! Hear!

E.N. KAUFMANN

\section{Physics and Society 17 (1) (January 1988) p. 10.}

2. Associating world wars with scientific disciplines tempts us to extrapolate. World War III will likely bring more glory to computer science than ever anticipated when video games first arrived. And given that III will be fought with the unprecedented destructive power now at our disposal, we propose geology as the preeminent discipline for World War IV

3. Physics Through the 1990s, Committee on the Physics Survey of the National Research Council (National Academy Press, Washington DC, 1986).

4. MRS BULLETIN Vol. XIII No. 5, p. 41-42.

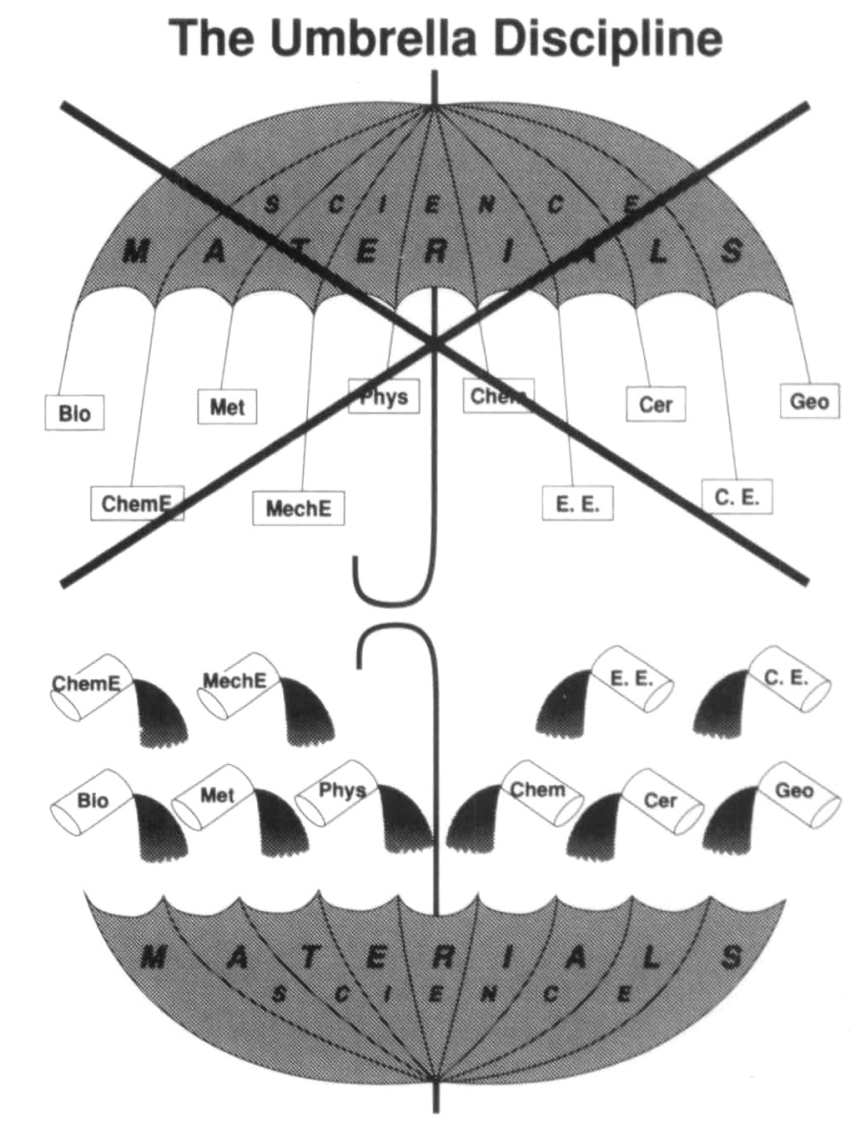

To characterize materials science as a super-discipline encompassing portions of most "classical" disciplines not only offends those dedicated to maintenance of their disciplinary identity but it is also not correct. Neither is materials science a subdiscipline of other fields. Rather, it benefits, from, and is in fact the product of, contributions of expertise from those trained in a variety of specific areas notwithstanding what they call themselves. Territorial imperatives need not apply to the field which is our joint creation. 